\title{
Modelización de la energía absorbida en la fusión de un metal por un haz láser ${ }^{(\bullet)}$
}

\author{
J. Oñoro ${ }^{(*)}$, R. Bermejo ${ }^{(* *)}$, A. Portolés ${ }^{(*)}$ y R. Gamboa ${ }^{(*)}$ \\ Resumen La interacción de un haz láser de alta densidad de energía con una superficie metálica puede producir \\ la formación de una zona fundida estrecha y profunda de geometría característica en keyhole (ojo de \\ llave). La cantidad de energía absorbida por la pieza será función de los parámetros de ejecución del \\ láser incidente y de la naturaleza y geometría de la pieza. En este trabajo, se presenta una formula- \\ ción que permite determinar cuantitativamente la cantidad de energía absorbida por una superficie \\ metálica, en función de una serie de parámetros experimentales. La formulación desarrollada tiene \\ especial aplicación en los ensayos de refusión, muy utilizados para analizar las interacciones láser- \\ metal, en los trabajos de investigación con tecnología láser. La formulación se ha particularizado \\ para dos aleaciones de aluminio de los grupos Al-Mg-Si y Al-Zn-Mg.
}

Palabras clave: Láser. Modelización. Láser-metal. Énsayos de refusión. Aleaciones de aluminio.

\section{Modelling the energy absorbed during a laser beam metal melting process}

\begin{abstract}
The interaction between a high-density laser beam and a metallic surface produces a deep and narrow melted area that has a characteristic keyhole geometric pattern. The amount of energy absorbed by the metal will depend on the parameters of the laser beam being applied, and the nature and geometry of the piece. In this paper, a formulation is presented which makes it possible to quantitatively determine the amount of energy absorbed by the metal as a function of a series of experimental parameters. The formulation that has been developed is especially applicable in beadon-plate tests commonly used to analyze laser-metal interactions in laser technology research. The formulation applies specifically to two types of aluminium alloys, $\mathrm{Al}-\mathrm{Mg}-\mathrm{Si}$ and $\mathrm{Al}-\mathrm{Zn}-\mathrm{Mg}$.
\end{abstract}

Keywords: Laser. Modelling. Laser-metal. Bead-on-plate tests. Aluminium alloys.

\section{INTRODUCCIÓN}

El rayo láser (light amplification by stimulated electromagnetic radiation) es un rayo de luz coherente y monocromático de alta densidad de energía, es decir, una radiación fotónica concentrada de una única longitud de onda, la cual depende del sistema utilizado para su generación, $0,69 \mu \mathrm{m}$ en el láser de rubí, $1,06 \mu$ en el láser de Nd-YAG ó 10,6 $\mu \mathrm{m}$ en el láser de $\mathrm{CO}_{2}(1-3)$.

La tecnología del tratamiento de materiales con láser se basa en la concentración de este rayo de luz

$(\bullet$ Trabajo recibido el día 7 de noviembre de 1997 y aceptado en su forma final el 24 de abril de 1998.

(*) Dpto. Ingeniería y Ciencia de los Materiales. E.T.S.I Industriales. Universidad Politécnica de Madrid. c/ José Gutiérrez Abascal, 2. 28006-Madrid (España).

(**) Centro Español de Soldadura (CESOL). c/ San Bernardo 123. 28015-Madrid (España). sobre la superficie del material a tratar, donde se transforma la energía fotónica en energía térmica, produciendo un aumento en la temperatura de la superficie del material.

En el tratamiento de materiales metálicos, cuando la densidad de energía aportada es baja, se produce una transferencia de calor por conducción, que produce un aumento local de la temperatura de la superficie del material, que puede llegar a fundir, sin llegar a la vaporización. La cantidad de energía absorbida es baja, al producirse notables pérdidas por reflexión del haz (1 y 4). Si por el contrario, la densidad de energía es alta, alcanzando densidades de potencia iguales o superiores a $10^{5} \mathrm{~W} / \mathrm{cm}^{2}$, se produce una profunda penetración del láser dentro del metal, formando una columna llena de un gas metálico ionizado que absorbe la mayor parte de la energía emitida por el láser en un pequeño volumen; esta columna de vapor permanece rodeada por 
un baño líquido, cuya presión mantiene el metal fundido contra las paredes del orificio formado. $\mathrm{Al}$ mover el haz en relación a la pieza, el orificio se desplazará formando un canal, que en su parte delantera tendrá un frente de fusión, mientras que en la parte de atrás se irá rellenando por la condensación y solidificación del metal, adoptando una forma característica denominada keyhole (ojo de llave) (1-3).

La tecnología láser ha sido utilizada con éxito en el corte y la unión de una gran variedad de metales y aleaciones, como aceros al carbono y de baja aleación, aceros inoxidables, aluminio, plomo, titanio y aleaciones resistentes a temperatura elevada (1-3). Sin embargo, existen todavía muchos interrogantes en cuanto a los procesos de interacción láser-superficie metálica, así como una importante cantidad de metales y aleaciones donde la aplicación del láser requiere avances significativos para su aplicación industrial.

De la energía incidente de un haz láser sobre la superficie metálica, una parte de esta energía será absorbida por el material, mientras otra parte será reflejada. Si la energía absorbida es suficientemente elevada se producirá durante el proceso la evaporación de una pequeña fracción de material, a su alrededor una estrecha zona fundida, y más allá una zona afectada térmicamente causada por el calor transmitido por conducción.

La cantidad de energía absorbida en la interacción láser-metal depende de una gran cantidad de factores entre ellos el tipo de metal o aleación, la geometría de la pieza, el acabado superficial, la naturaleza del haz láser, la potencia, la geometría del haz, la naturaleza del gas de protección, la focalización, etc. (1-4).

En los estudios de la interacción láser-metal, la realización de ensayos de refusión es una herramienta ampliamente utilizada para la evaluación del comportamiento de los materiales, de los parámetros de operación y de las condiciones de ensayo (57). Sin embargo, la evaluación de los resultados obtenidos durante estos ensayos es de naturaleza cualitativa, ya que los resultados obtenidos deben expresarse en función de la geometría, el aspecto superficial, etc, de las muestras ensayadas.

En este trabajo se presenta una formulación que mediante la obtención de las áreas de las zonas fundida y vaporizada, junto con la profundidad máxima de la zona fundida desde la superficie, se puede calcular directamente la cantidad de energía absorbida por la muestra, permitiendo su cuantificación. La formulación presentada es general para cualquier tipo de metal o aleación. Se ha realizado una serie de simplificaciones, para la resolución de las ecuaciones diferenciales planteadas, de acuerdo con una serie de hipótesis. Se ha realizado una aplicación del modelo a dos aleaciones de aluminio
AA6082 (Al-Mg-Si) y AA7020 (Al-Zn-Mg); los valores de las constantes físicas se han particularizado para dichas aleaciones.

El aluminio y sus aleaciones son materiales difíciles de fundir con láser. El pobre acoplamiento del haz sobre la superficie del aluminio es debido en parte a la alta densidad de electrones libres en el sólido (responsables de su alta conductividad térmica y eléctrica), haciendo del aluminio uno de los mejores reflectores de la luz (8). A este efecto hay que añadirle la formación de un plasma bloqueante del haz cuando en la composición de la aleación de aluminio existen elementos tales como el magnesio y el zinc, de baja temperatura de vaporización (7).

La energía asociada a un fotón de un láser de $\mathrm{CO}_{2}(10,6 \mu \mathrm{m})$ es de $0,1 \mathrm{eV}$, baja en comparación con la de un electrón de un haz de electrones, del orden de $100.000 \mathrm{eV}$. Por esta razón, el haz láser tiene una gran tendencia a interaccionar con los electrones libres existentes en el plasma (gas de protección y vapor metálico formado por la incidencia del haz sobre el aluminio). Este plasma, en el caso del aluminio, tiende a dispersar parte del haz (efecto desfocalizador), dando lugar a una interacción inestable, con zonas fundidas anchas y poco profundas, comparadas con las obtenidas con otros materiales, y un aspecto superficial irregular (3).

\section{FORMULARIO Y UNIDADES}

$q_{\mathrm{V}}$. Energía suministrada por unidad de tiempo y unidad de volumen, $\mathrm{W} / \mathrm{m}^{3}$.

$q_{\mathrm{i}}$. Energía incidente por unidad de tiempo, W.

$q_{\mathrm{C}}$. Energía transmitida por conducción por unidad de tiempo, W.

$q_{\mathrm{Ab}}$. Energía absorbida por unidad de tiempo, W.

$A$ Porcentaje de energía absorbida por la superficie metálica.

$t$ Tiempo, s.

$T$ Temperatura, K.

$T_{0} \quad$ Temperatura inicial del material, $\mathrm{K}$.

$T_{\mathrm{m}} \quad$ Temperatura de fusión del material, $\mathrm{K}$.

$v \quad$ Velocidad de desplazamiento de la pieza, $\mathrm{m} / \mathrm{s}$.

$r_{\mathrm{B}} \quad$ Radio del haz láser, $\mathrm{m}$.

$\lambda \quad$ Conductividad térmica, $\mathrm{W} / \mathrm{mK}$.

$t \quad$ Tiempo desde la incidencia del haz, s.

$t_{0} \quad$ Tiempo que tarda el calor en difundir una distancia $r_{\mathrm{B}}$; es decir, la mitad de la longitud de la fuente lineal, $s$.

$a \quad$ Difusividad térmica del material, $\mathrm{m}^{2} / \mathrm{s}$.

$Z_{0} \quad$ Profundidad que el calor recorre durante la interacción del haz, $\mathrm{m}$.

$Z_{\mathrm{m}}$. Profundidad de la zona fundida, $\mathrm{m}$.

$Z_{\mathrm{t}} \quad$ Espesor del material, $\mathrm{m}$.

$\rho$ Densidad, $\mathrm{kg} / \mathrm{m}^{3}$.

C Calor específico, $\mathrm{J} / \mathrm{kgK}$ 
$A_{\mathrm{m}} \quad$ Área fundida, $\mathrm{m}^{2}$.

$A_{\mathrm{V}} \quad$ Área vaporizada, $\mathrm{m}^{2}$.

$L_{\mathrm{m}} \quad$ Calor latente de fusión, $\mathrm{J} / \mathrm{kg}$.

$L_{\mathrm{V}} \quad$ Calor latente de vaporización, $\mathrm{J} / \mathrm{kg}$.

\section{FORMULACIÓN DEL MODELO}

La energía absorbida en la interacción lásermetal, durante un instante cualquiera del proceso de fusión, puede dividirse en:

- Energía transmitida por conducción.

- Energía absorbida en la fusión y la vaporización.

- Energía emitida por radiación al medio circundante.

\subsection{Energía transmitida por conducción}

La modelización del proceso de transmisión de calor durante la interacción láser-metal se supondrá que se realiza con formación de una columna de plasma que penetra en el interior de la pieza según aparece en la figura 1. En estas condiciones, cuando una fuente de calor incide sobre la superficie de un sólido, el campo de temperatura generado en su interior debe satisfacer las ecuaciones de la transmisión de calor, que en el caso de considerar que las propiedades físicas del material no varían con la temperatura, las condiciones de transmisión de calor a lo largo de la pieza vendrán regidas por la ecuación diferencial:

$$
\nabla^{2} T-\frac{1}{a} \frac{\mathrm{d} T}{\mathrm{~d} t}+\frac{q_{\mathrm{v}}}{\lambda}=0
$$

La solución de esta ecuación diferencial, para un haz de energía que se desplaza con una velocidad $v$ sobre la superficie del material, permite obtener, tras la resolución de complicadas integrales múltiples, los valores de la temperatura en cada instante para cada punto del material (9 y 10).

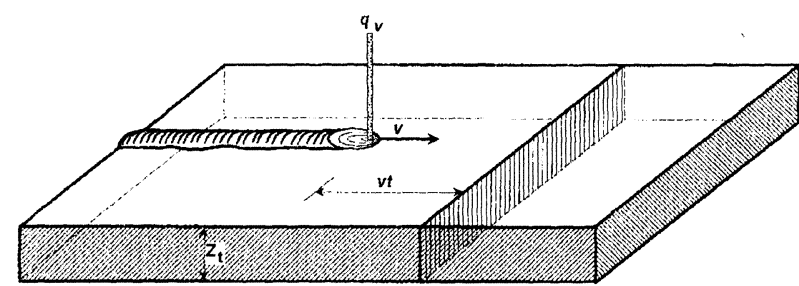

Fig. 1.- Esquema de la realización de un ensayo de refusión de un haz láser que aporta una energía, $q_{\mathrm{V}}$, y se desplaza sobre la muestra a una velocidad $v$.

FIG. 1.- Schematic of a bead-on-plate test where a laser beam supplies power $\mathrm{q}_{\mathrm{v}}$ and travel through the sample to speed $\mathrm{v}$.
La utilización del modelo de Ashby-Easterling (10) permite obtener una solución analítica aproximada, mediante la aplicación de una serie de condiciones de contorno que facilitan la resolución de la ecuación diferencial. El modelo de Ashby-Easterling proporciona una solución analítica aproximada del campo de temperaturas en una sección determinada de la pieza (Fig. 1). Al considerar que la velocidad del proceso es suficientemente elevada, la fuente del haz láser con modo de geometría gausiana (TEM 00)(Transverse Electromagnetic Mode) (Fig. 2) puede idealizarse como una línea de espesor finito en la coordenada, $y$, pero infinitesimal en la dirección del movimiento, $x$.

El campo de temperaturas para cualquier punto dentro del haz láser puede ahora representarse por la ecuación

$$
T-T_{0}=\frac{A \cdot q_{\mathrm{i}} / v}{2 \pi \lambda\left(t\left(t+t_{0}\right)\right.} \exp -\left(\frac{\left(Z+Z_{0}\right)^{2}}{4 \mathrm{a} t}\right)
$$

donde la constante $t_{0}$ mide el tiempo que tarda el calor en recorrer una distancia igual al radio del haz, $r_{\mathrm{B}}^{2} / 4 a$, y la longitud $Z_{0}$ mide la distancia que el calor recorre durante el tiempo de interacción del haz, $r_{\mathrm{B}} / \nu$.

En el campo de temperaturas, la temperatura máxima se alcanzará donde $\mathrm{d} T / \mathrm{d} t=0$. Y en el límite cuando $t<<t_{0}$

$$
T-T_{0}=\frac{2 A \cdot q_{\mathrm{i}} / v}{e \pi \rho C\left(Z+Z_{0}\right)^{2}}
$$

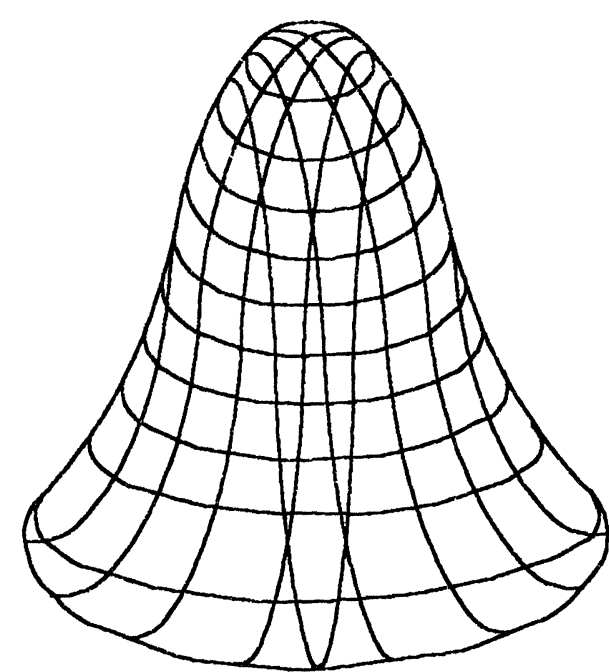

FIG. 2.- Distribución gausiana de energía del haz láser utilizado. TEM 00.

FIG. 2.- Energy gaussian distribution of laser beam used. TEM 00. 
Para el punto en la vertical del haz láser en que acaba la zona fundida $Z=Z_{\mathrm{m}}$, la temperatura del baño de fusión será igual a la temperatura de fusión de la aleación $T=T_{\mathrm{m}}$, donde despejando se puede obtener la fracción de calor absorbida que produce por conducción un gradiente térmico causado por un haz láser que se desplaza a una velocidad, $v$ :

$$
A \cdot q_{i}=q_{c}=\frac{\left(T_{\mathrm{m}}-T_{0}\right) e \pi \rho C\left(Z_{\mathrm{m}}+Z_{0}\right)^{2}}{2} v
$$

Si en esta ecuación, consideramos que el flujo térmico alcanza una profundidad que será función del espesor del material ensayado y la profundidad de la zona fundida (Fig. 3), se puede sustituir el valor de $Z_{0}$ por la relación:

$$
Z_{0}=\left(Z_{\mathrm{t}}-Z_{\mathrm{m}}\right)\left(Z_{\mathrm{m}} / Z_{\mathrm{t}}\right)^{2}
$$

Esta simplificación permite obtener el valor del flujo térmico en función de parámetros experimentales, geométricos y de las propiedades físicas del material ensayado.

\subsection{Energía absorbida en la fusión y la vaporización}

Además de la energía transmitida existe una porción de la energía incidente que es absorbida por la fracción de material que se funde y vaporiza. Aunque esta energía se libera durante el posterior enfriamiento, temporalmente es sustraida del aporte energético instantáneo, por lo que no es aprovechable para la fusión de más material, y tampoco está considerada dentro de la fracción de energía absorbida por conducción, ya que en la simplificación realizada se considera que toda la zona fundida se

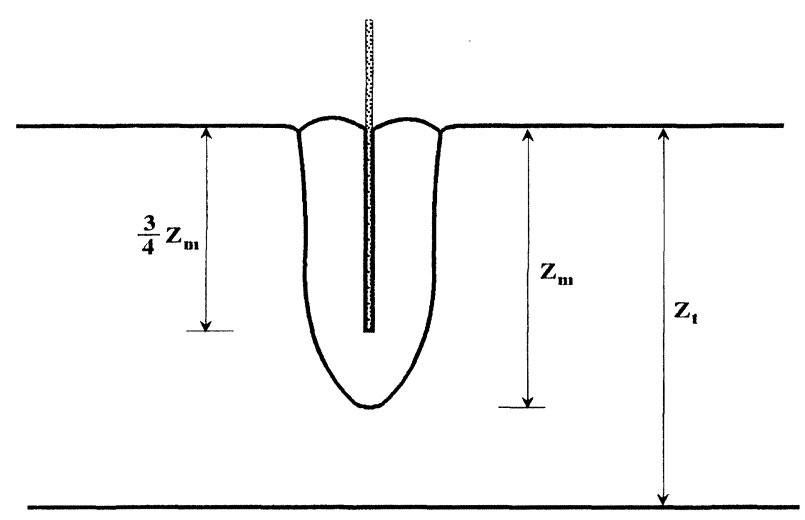

FIG. 3.- Geometría de las zonas fundidas y vaporizadas en relación con el espesor de la pieza.

FIG. 3.- Melted and vaporized areas geometry in relation to the piece thickness. encuentra a la temperatura de fusión de la pieza. Por tanto, esta energía es absorbida por el material y debe ser evaluada.

$$
q_{\mathrm{Ab}}=\left(A_{\mathrm{m}} L_{\mathrm{m}}+A_{v} L_{v}\right) v \rho
$$

donde el área fundida puede ser medida experimentalmente, y el área vaporizada se calcula a partir del radio de la columna de plasma y la profundidad de la zona fundida (Fig. 3), suponiendo que:

$$
A_{\mathrm{v}}=2 r_{\mathrm{B}} 3 Z_{\mathrm{m}} / 4
$$

El valor de $r_{\mathrm{B}}$ será función de las características del equipo, del material y de los parámetros utilizados en el procedimiento efectuado, debiendo calcularse o estimarse durante la realización de los ensayos para cada aplicación. De los demás parámetros utilizados en la modelización, unos son valores físicos dependientes del material y el resto se obtienen experimentalmente de las muestras ensayadas.

\subsection{Energía emitida por radiación al medio circundante}

Las emisiones por radiación se pueden evaluar a partir de la ley de Stefan-Boltzmann, estas emisiones sólo son significativas a temperaturas muy elevadas; en este caso, en las zonas fundidas y vaporizadas, las cuales presentan un área de radiación extremadamente reducida. Las estimaciones realizadas de las pérdidas por radiación en la interacción láser-metal indican valores muy bajos (11), por lo que se consideran despreciables en esta modelización.

\section{APLICACIÓN DEL MODELO A DOS ALEACIONES DE ALUMINIO}

Se han seleccionado dos aleaciones de aluminio de los grupos Al-Mg-Si (AA6082) y Al-Zn-Mg (AA7020) (Tabla I). Ambas aleaciones son tratables térmicamente por bonificado y se consideran soldables mediante procesos convencionales de soldadura. La aleación Al-Mg-Si tiene un bajo contenido de elementos de aleación. Por otro lado, la aleación $\mathrm{Al}-\mathrm{Zn}-\mathrm{Mg}$ tiene un mayor contenido de elementos de aleación, especialmente de bajo punto de fusión, que son los que presentan mayores dificultades en la interacción láser-aluminio.

La aplicación del modelo propuesto, para analizar la energía absorbida por una aleación de aluminio, a partir de su interacción con el haz láser, necesitará la definición de una serie de hipótesis y parámetros de ejecución que estén en concordancia con los planteamientos del modelo. 
TABla I.- Composición química de las aleaciones modelizadas

TABLE I.- Chemical compositions of modelling alloys

\begin{tabular}{|c|c|c|c|c|c|c|c|c|c|}
\hline Aleación & $\mathrm{Si}$ & $\mathrm{Fe}$ & $\mathrm{Cu}$ & $\mathrm{Mn}$ & $\mathrm{Mg}$ & $\mathrm{Cr}$ & $\mathrm{Zn}$ & $\mathrm{Ti}$ & $\mathrm{Al}$ \\
\hline AA-6082 & 1,05 & 0,5 & 0,1 & 0,7 & 0,9 & 0,25 & 0,2 & 0,1 & 96,2 \\
AA-7020 & 0,35 & 0,4 & 0,2 & 0,3 & 1,2 & 0,25 & 4,5 & 0,15 & 92,7 \\
\hline
\end{tabular}

\subsection{Hipótesis del modelo}

- Velocidad del proceso $1 \mathrm{~m} / \mathrm{min}$, suficientemente elevada para considerar que la fuente de calor se localiza sobre la superficie de la probeta.

- Distribución gausiana de la densidad de potencia (TEM 00).

- Diámetro de la columna de plasma de 0,25 mm.

- Se consideran constantes las propiedades físicas de las aleaciones de aluminio utilizadas. En los cálculos se utilizarán valores promediados.

- Las pérdidas de calor por convección y radiación en la superficie de la muestra se consideran despreciables frente a la disipación de calor por conducción.

- El calor latente de fusión y vaporización se calculan experimentalmente a partir del volumen de masa fundida y vaporizada.

\subsection{Resultados de la modelización}

Los ensayos para la aplicación del modelo se han realizado sobre muestras de las aleaciones AA6082 y AA7020 de dimensiones $200 \times 200 \times 4$ $\mathrm{mm}$, la capa de óxido fue eliminada antes de cada ensayo con cepillo de acero inoxidable. Los parámetros del haz láser utilizados fueron idénticos para ambos materiales (Tabla II).

La potencia real suministrada por el haz láser se ha determinado experimentalmente a través de un calorímetro instalado a la salida del láser.

Una vez realizados los ensayos de refusión, se procedió a seccionar las muestras en su parte central y se prepararon las secciones metalográficamente. La profundidad de la zona fundida se determinó en un proyector de perfiles, y el área fundida midiendo la superficie por análisis de imagen. Los valores calculados, así como los de las constantes físicas y el resto de parámetros utilizados en la modelización, se han recogido en la tabla III.

La aplicación del modelo a los valores obtenidos ha permitido el cálculo de las energías absorbidas en el proceso para ambas aleaciones (Tabla III).
TABLA II.- Parámetros del haz láser utilizados en los ensayos de refusión

TABLE II.- Laser beam parameters used on beadon-plates testing

\begin{tabular}{|l|c|}
\hline Equipo láser & RS-6000 \\
Modo & TEM 00 \\
Potencia nominal, $\mathrm{W}$ & 2000 \\
Potencia incidente, $\mathrm{W}$ & 1700 \\
Longitud focal, mm & 150 \\
Punto focal, mm & -1 \\
Boquilla-pieza, mm & 5 \\
Gas de protección & $\mathrm{Ar}$ \\
Caudal de gas, $\mathrm{L} / \mathrm{min}$ & 15 \\
Velocidad de soldeo, $\mathrm{m} / \mathrm{min}$ & 1 \\
\hline
\end{tabular}

\section{DISCUSIÓN}

El desarrollo de un modelo que integra tanto los aspectos de la energía absorbida por conducción

TABLA III.- Propiedades físicas y parámetros experimentales calculados en la modelización láseraluminio

TABLE III.- Physical properties and experimental parameters obtained in the laser-aluminium modelling

\begin{tabular}{|c|c|c|}
\hline Aleación & AA-6082 & AA-7020 \\
\hline$\rho, \mathrm{kg} / \mathrm{dm}^{3}$ & 2,7 & 2,77 \\
$T_{\mathrm{m}},{ }^{\circ} \mathrm{C}$ & 655 & 650 \\
$C, \mathrm{~J} / \mathrm{kgK}$ & 896 & 875 \\
$L_{\mathrm{m}}, \mathrm{kJ} / \mathrm{kg}$ & 386 & 384 \\
$L_{\mathrm{v}}, \mathrm{kJ} / \mathrm{kg}$ & 11.000 & 11.000 \\
$\nu, \mathrm{m} / \mathrm{min}$ & 1 & 1 \\
$T_{0},{ }^{\circ} \mathrm{C}$ & 20 & 20 \\
$Z_{\mathrm{m}}, \mathrm{mm}$ & 1,98 & 1,32 \\
$Z_{0}, \mathrm{~mm}$ & 4 & 4 \\
$A_{\mathrm{m}}, \mathrm{mm}{ }^{2}$ & 3,21 & 1,65 \\
$r_{\mathrm{B}}, \mathrm{mm}$ & 0,25 & 0,25 \\
$A_{\mathrm{v}}, \mathrm{mm}{ }^{2}$ & 0,37 & 0,25 \\
$q_{\mathrm{i}}, \mathrm{W}$ & 1.700 & 1.700 \\
$q_{\mathrm{c}}, \mathrm{W}$ & 670 & 282 \\
$q_{\mathrm{Ab}}, \mathrm{W}$ & 240 & 155 \\
$q_{\mathrm{total}}, \mathrm{W}$ & 910 & 437 \\
$q_{\mathrm{i}}, \%$ & 54 & 26 \\
\hline
\end{tabular}

Rev. Metal. Madrid, 34 (5), 1998425

http://revistademetalurgia.revistas.csic.es 
como la existencia de una cantidad adicional de energía que inicialmente se concentra en la zona fundida, para luego transmitirse al resto del material, permite un análisis global del fenómeno que se produce en la interacción láser-metal. Por otro lado, la utilización de una formulación simplificada permite que el modelo pueda aplicarse fácilmente a los procesos de interacción láser-superficie metálica y, especialmente, para los ensayos de refusión sobre aleaciones de aluminio, para los que ha sido aplicado.

El modelo evalúa el proceso de absorción de calor por el material a través de dos procesos que se superponen en el tiempo. Por un lado, mediante la modificación del modelo de Ashby-Easterling para la transmisión de calor durante la realización de uniones soldadas (12), se ha calculado la absorción de calor por conducción de un cuerpo frío, en cuyo interior existe un foco de calor a temperatura constante. Y por otro, se ha obtenido la absorción de calor debida a los procesos locales de fusión y vaporización que se producen en la zona de interacción del láser con el material.

La aplicación del modelo de Ashby-Easterling con las simplificaciones señaladas anteriormente permite la evaluación de la energía absorbida a través de una formulación donde únicamente intervienen constantes físicas del material y valores que pueden calcularse experimentalmente.

La cuantificación de la energía absorbida por conducción supone la existencia de un foco caliente a la temperatura de fusión del material base, que produce un gradiente de temperaturas en las inmediaciones de dicho foco. La absorción de calor desde este foco caliente al resto del material, se realiza a través de un proceso de conducción térmica unidireccional. La temperatura del foco caliente no es constante, sino que varía desde la zona de contacto entre el sólido y el líquido, que está a la temperatura de fusión, hasta valores mucho más altos, en el resto de la zona fundida y vaporizada, donde se dan las condiciones de plasma. Sin embargo, la interfase sólido-líquido es la superficie que genera la transmisión de calor por conducción al resto del material que se encuentra en estado sólido, y esta interfase tiene durante todo el proceso una temperatura muy próxima a la de fusión del material. Al haber dividido el proceso de absorción en dos procesos diferentes, la evaluación del calor absorbido en la fusión y la vaporización de la zona fundida, tienen en cuenta este calor absorbido por el material, que no está recogido en la otra parte del modelo.

En esta aplicación de la formulación de AshbyEasterling, se ha realizado la estimación de la profundidad que alcanza el flujo térmico, como un parámetro dependiente del espesor del material ensayado, y de la profundidad de la zona fundida, obtenida tras el ensayo de valor

$$
Z_{0}=\left(Z_{\mathrm{t}}-Z_{\mathrm{m}}\right)\left(Z_{\mathrm{m}} / Z_{\mathrm{t}}\right)^{2}
$$

esta estimación, al considerar la geometría del proceso dentro de la formulación, permite unificar en un solo modelo la posible utilización de chapas más o menos gruesas, o la mayor o menor penetración de la zona fundida dentro de la pieza. De tal forma que cuando la zona fundida abarca casi todo el espesor de la pieza, el modelo identifica como fuente de calor prácticamente todo el espesor de la pieza, mientras que cuando la zona fundida se reduce a un pequeño porcentaje del espesor, la dimensión de la fuente de calor también se reduce significativamente, en función de la relación entre las dimensiones relativas del espesor total y la profundidad de la zona fundida.

El calor absorbido en la fusión y la vaporización, durante la interacción láser-metal, permanece absorbido en la zona fundida y vaporizada, que actúa como foco caliente en el proceso de transmisión de calor; posteriormente, cuando el haz láser se va desplazando de un punto a otro del material, este calor es cedido mayoritariamente al resto de la muestra. La modelización de esta energía se ha realizado considerando el volumen de material fundido y vaporizado obtenido a partir de valores experimentales.

La cantidad de volumen vaporizado se ha obtenido a partir de la profundidad de la zona fundida y del radio de la columna de plasma formada durante el ensayo. La geometría de esta zona se ha supuesto cilíndrica durante todo el proceso para simplificar la formulación. La geometría de la columna de plasma, en la superficie de las muestras, se ha obtenido mediante pirómetro óptico, durante la realización de los ensayos, siendo las dimensiones obtenidas similares a las calculadas por otros investigadores (13 y 14). El valor del área vaporizada, aunque cuantitativamente es muy pequeña en relación al área fundida, tiene influencia en el cálculo de la energía absorbida, debido al elevado valor del calor latente de vaporización. La obtención experimental del volumen de material vaporizado puede presentar cambios significativos en función de los parámetros del haz láser utilizado y la naturaleza del material ensayado (13). La generalización del modelo a otros materiales y condiciones de ejecución, requerirá una evaluación de este parámetro, que no podrá aplicarse directamente con otros parámetros de ejecución o con otros materiales.

Las propiedades físicas de las aleaciones estudiadas se han considerado constantes para simplificar el modelo. Al utilizar valores promedio de los que se verifican en el intervalo de temperaturas donde tiene lugar el proceso, la aproximación realizada no representa una modificación significativa de los valores reales. 
Los parámetros experimentales obtenidos durante los ensayos, así como los valores de los porcentajes de energía absorbida obtenidos a partir del modelo (Tabla III), son similares a los obtenidos por otros investigadores $(5,7,11$ y 15$)$, donde se han utilizado otros sistemas de evaluación en procesos de interacción láser-aluminio.

Los valores de absorción, en las dos aleaciones estudiadas (Tabla III), recogen diferencias importantes entre ambas aleaciones, tanto del área como de la profundidad máxima de la zona fundida. Las diferencias entre las constantes físicas de ambos materiales, por sí mismas no conducen a valores de energía absorbida muy distintos. Estos resultados experimentales tan distintos se deben al mayor contenido de elementos de bajo punto de ebullición ( $\mathrm{Zn}$ y Mg) en la aleación AA 7020, que como se mencionó anteriormente, produce un plasma bloqueante del haz que reduce la absorción de la energía incidente.

\section{CONCLUSIONES}

- Se ha modelizado la energía absorbida en la interacción láser-metal, donde se han incluido aspectos de la geometría de la pieza, la velocidad de ejecución del proceso y parámetros obtenidos experimentalmente dependientes de la geometría de la zona fundida.

- La modelización de la energía absorbida se ha realizado considerando dos procesos diferentes. Por un lado, un proceso de absorción de calor por conducción a partir de un foco de calor puntual a temperatura constante, utilizando una simplificación de la formulación de Ashby-Easterling. Por otro lado, un proceso de absorción de calor debido a la fusión y evaporación de la aleación dentro de la zona fundida.

- El modelo desarrollado es sencillo y de fácil aplicación, por lo que puede utilizarse en el análisis y cuantificación de ensayos de refusión en la interacción láser-metal.
- La aplicación del modelo en dos aleaciones de aluminio ha obtenido valores de absorción similares a los obtenidos en estos materiales por otros métodos de evaluación.

- El modelo ha sido aplicado para dos aleaciones de aluminio, pero al ser su formulación genérica puede utilizarse, con pequeñas modificaciones, en la interacción de cualquier otro material metálico con un haz láser.

\section{Agradecimiento}

Los autores agradecen a la Comisión de Investigación Científica y Técnica (CICYT), la financiación concedida al proyecto (MAT94-0640-C02-01) que ha permitido la realización de este trabajo.

\section{REFERENCIAS}

(1) Steen, W.M. Laser Material Processing. Springer-Verlag. Londres, 1991: 12, 109, 114 y 136.

(2) Craferand, R.C. y OAKLeY, P.J. Laser Processing in Manufacturing. Chapman \& Hall. Londres, 1993: 19-44.

(3) Mazumder, J. J. Met. 34 (Jul), 1982: 16-24.

(4) SwIfT-Hook, D.T. y Gick, A.E.F. Weld. Res. (Nov.) 1973: 492s-499s.

(5) Matsumura, H. et al. Proc. LAMP 92. (Jun) 1992: 529533.

(6) KIM, J.S. et al. Proc. ICALEO 93. 1993: 640-650.

(7) Huntington, C.A. y Eagar, T.W. Weld. Res. (Abril) 1983: 105s-107s.

(8) Davis, J.R. Aluminum and Aluminum Alloys. Ed. ASM International. Materials Park, OH. 1994: 644.

(9) Rosenthal, D. Weld. J. 20 (5), 1941: 220s-234s.

(10) Ashby, M.F. y EAsterling, K.E. Acta Metall. 32 (11), 1984: 1935-1948.

(11) Chun, M.K. y Rose, K.J. Appl. Phys. 41 (2), 1970: 614 620.

(12) Ashby, M.F. y Easterling, K.E. Acta Metall. 30 (11), 1982: 1969-1978.

(13) Davis, M. et al. Weld. Res. (Jul) 1986: 167s-174s.

(14) VALDEZ, D. et al. Rev. Soldadura. 21 (1), 1991: 3-18.

(15) Malin, V. Weld. Res. (Sept) 1995: 305s-318s. 